\title{
Разработка и валидация биоаналитической методики количественного определения иматиниба в плазме крови человека методом ВЭЖХ
}

\author{
Леонов К.А. ${ }^{1}$, Бакибаев А.А. ${ }^{2}$ \\ ${ }^{1}$ ФАОУ ВО Наџиональный исследовательский Томский политехнический университет, Томск \\ ${ }^{2}$ ФГАОУ ВО Наџиональный исследовательский Томский государственньй университет, Томск
}

Поступила в редакцию 05.05.2016 г.

\begin{abstract}
Разработана новая высокочувствительная биоаналитическая методика количественного определения иматиниба в плазме крови человека методом ВЭЖХ с УФ-детектированием. Для выделения иматиниба из биологической матрицы использована жидкостно-жидкостная экстракция ацетонитрилом по принципу QuEChERS. Хроматографический анализ проводили на приборе Милихром A-02 co спектрофотометрическим детектированием при 260 нм. В качестве подвижной фазы использовали 0.5 \% раствор калия дигидрофосфата - метанол - вода 74:25:1 с $\mathrm{pH}$ равным 3.3 и метанол с $\mathrm{pH}$ равным 3.3. Путем валидации доказана пригодность разработанной биоаналитической методики для различных фармакокинетических исследований.
\end{abstract}

Ключевые слова: иматиниб, плазма крови, ВЭЖХ, валидация.

\section{Development and validation of bioanalytical technique of imatinib quantitative determination in human plasma by HPLC}

\author{
Leonov K.A. ${ }^{1}$, Bakibaev A.A. ${ }^{2}$ \\ IFSAEU of HE National Research Tomsk Polytechnic University, Tomsk \\ 2FSAEU of HE National Research Tomsk State University, Tomsk
}

Imatinib is one of the best anticancer drugs used in the treatment of melanoma, and myeloleukemia. According to federal program "PHARMA 2020", involving the import substitution of drugs, imatinib is of great interest to pharmaceutical manufacturers. For state registration of generics in accordance with the Russian legislation is necessary to confirm its efficacy and safety of the original drug through a clinical bioequivalence studies. A mandatory step in this research is the development of analytical methods for quantitative determination of the drug in human plasma with a preliminary selection of sample preparation conditions. Currently, according to literature data, the determination of imatinib in human blood plasma by HPLC with UV detection has at least $50 \mathrm{ng} / \mathrm{ml}$ limit of detection. In this context, the aim of this work was to develop a more sensitive bioanalytical method of imatinib quantitative determination in human plasma by HPLC with UV detection and its validation.

Quantitative determination of imatinib in plasma was carried out on a chromatograph MilichromA02 (CJSC "EcoNova", Novosibirsk) column size $75.0 \times 2.0 \mathrm{~mm}$ filled with 5 micron sorbent ProntoSIL $\mathrm{C}_{18}$ and spectrophotometric detection at $260 \mathrm{~nm}$. To reduce the limit of quantification of imatinib in plasma and, hence, improve sensitivity of the test, compared with the already known data, applied dynamic modification of the mobile phase. A mixture of $0.5 \%$ potassium dihydrogen phosphate, methanol and triethylamine solution was used as mobile phase in the ratio 74:25:1 at a pH of 3.3, adjusted with orthophosphoric acid, as mobile phase $\mathrm{B}$ used methanol with a $\mathrm{pH}$ of 3.3. Eluent flow rate was $0.1 \mathrm{ml} / \mathrm{min}$, column temperature $-35^{\circ} \mathrm{C}$, sample injection volume - 20 microliters. Method liquid-liquid extraction principle acetonitrile QuEChERS was used for extraction from plasma. 
To confirm the suitability of the developed method was performed a validation on the basis of its «Guidelines for the examination of drugs. Volume 1» (Russia, 2013) and in accordance with the validation guidelines requirements « Guidance for Industry bioanalytical methods: Bioanalytical method validation» (FDA, USA, 2001) and «Guideline on validation of bioanalytical methods» (EMA, England, 2009). Selectivity of techniques and its linearity in the concentration range from 42 to $4200 \mathrm{ng} / \mathrm{ml}$ were proved. Accuracy of the procedure ranges is from 92.1 to $104.7 \%$, and its precision - from 92.6 to $97.9 \%$. The absence of the transfer of an analyte in a sample after the sample single analysis with a high concentration of analyte is demonstrated, stability of analyzed samples is elicited.

Developed and validated technique can be applied to the analysis of human plasma samples containing imatinib in various pharmacokinetic studies and clinical bioequivalence study.

Keywords: imatinib, blood plasma, HPLC, validation.

\section{Введение}

Иматиниб является одним из лучших противоопухолевых препаратов, применяемых в таргетной (целевой) терапии при меланоме и миелолейкозе. Он ингибирует протеинтирозинкиназу, селективно подавляя пролиферацию и вызывая апоптоз клеточных линий, а также незрелых лейкозных клеток [1]. Структурная формула иматиниба приведена на рис. 1.

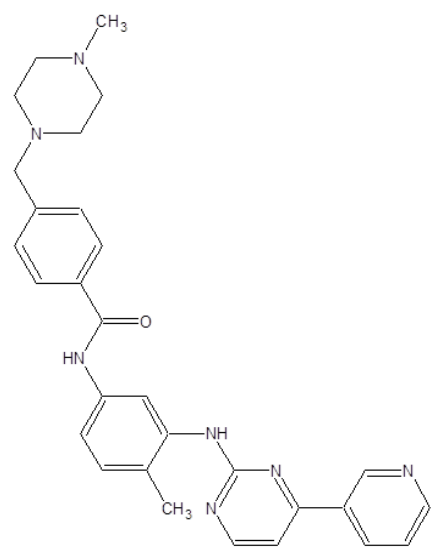

Рис. 1. Структурная формула иматиниба

В связи с федеральной программой «ФАРМА-2020», предполагающей импортозамещение лекарственных средств, иматиниб представляет огромный интерес для фармацевтических производителей. Для государственной регистрации дженерика в Российской Федерации согласно Российскому законодательству необходимо подтверждение его эффективности и безопасности оригинальному препарату, которое устанавливается путем клинического исследования биоэквивалентности. Обязательным этапом на пути проведения такого исследования является разработка аналитической методики количественного определения лекарственного средства в плазме крови с предварительным подбором условий выделения исследуемого вещества из биологической среды [2-3].

Основными методами определения иматиниба в плазме крови являются ВЭЖХ с УФ-детектированием [2-11] и ВЭЖХ с масс-спектрометрическим детектированием [7, 9, 12-15]. Метод ВЭЖХ с масс-спектрометрическим детектированием обладает высокой чувствительностью (около 5 нг/см ${ }^{3}$ ), однако является дорогостоящим. Основными преимуществами ВЭЖХ с УФ-детектированием являются более низкая стоимость в сравнении с ВЭЖХ-МС, простота и доступность, что позволяет применять его в большинстве фармакокинетических исследований. В настоящее время, согласно литературным данным, в случае определения этим методом имати- 


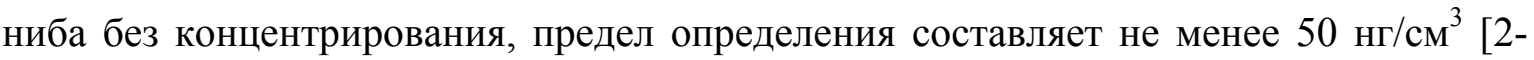
$11]$.

В связи с этим, целью данной работы являлась разработка более чувствительной биоаналитической методики количественного определения иматиниба в плазме крови человека методом ВЭЖХ с УФ-детектированием и ее валидация.

\section{Эксперимент}

Аппаратура. Количественное определение иматиниба в плазме крови проводили на хроматографе Милихром А-02 (ЗАО «ЭкоНова», г. Новосибирск) с УФдетектором. Хроматографическое разделение осуществляли на колонке размером $75 \times 2$ мм, заполненной сорбентом ProntoSIL C18, 5 мкм. Сбор и обработку данных выполняли при помощи программного обеспечения «Мультихром» (ЗАО «Амперсенд», Россия). В процессе подготовки проб использовали лабораторный встряхиватель Elmi V-3 (Латвия), лабораторную центрифугу Heraeus Biofuge pico (Германия) и вакуумный концентратор UniEquip Univapo 100 ECH (Германия).

Реактивы. В работе применяли образец субстанции иматиниба мезилата $\mathrm{C}_{29} \mathrm{H}_{31} \mathrm{~N}_{7} \mathrm{O} \cdot \mathrm{CH}_{2} \mathrm{SO}_{3} \mathrm{H}$ (Synthon Blansko, Чехия). Исходный раствор субстанции с концентрацией $1 \mathrm{мг} / \mathrm{cm}^{3}$ готовили растворением соответствующей навески в подвижной фазе А. В качестве биологической матрицы использовали плазму крови добровольцев, не принимавших препараты, содержащие иматиниб. Калибровочные растворы иматиниба в плазме крови готовили в день использования. Для приготовления подвижной фазы использовали метанол марки HPLC Grade (J.T.Baker, CША), ацетонитрил марки «для ВЭЖХ» (ООО «НПК Криохром», Россия), бидистиллированную воду (ГОСТ 7602-72), калий фосфорнокислый однозамещенный марки «хч» (Омскреактив, Россия), кислоту ортофосфорную марки «хч» (Россия), триэтиламин 99.9 \% (Panreac, США), натрия хлорид марки «хч» (Россия).

Пробоподготовка. Для извлечения иматиниба из плазмы крови использовали метод жидкостно-жидкостной экстракции ацетонитрилом по принципу QuEChERS (Quick, Easy, Cheap, Effective, Rugged, Safe). В центрифужную микропробирку вместимостью $2 \mathrm{~cm}^{3}$ помещали 500 мкл плазмы крови, содержащей иматиниб, прибавляли $1 \mathrm{~cm}^{3}$ ацетонитрила и тщательно перемешивали на встряхивателе Elmi V-3 при скорости 4000 об/мин в течение 10 мин. Затем к раствору прибавляли 0.1-0.2 г натрия хлорида и перемешивали в течение 5 мин. Полученный раствор центрифугировали при 13000 об/мин в течение 10 мин. Водную фазу отбрасывали, а органическую упаривали на вакуумном концентраторе при температуре $45^{\circ} \mathrm{C}$ в течение 80 мин. К сухому остатку прибавляли 500 мкл подвижной фазы А, перемешивали 5 мин и центрифугировали при 13000 об/мин в течение 5 мин. Надосадочную жидкость использовали для хроматографического анализа.

Таблица 1. Условия градиентного режима элюирования

\begin{tabular}{|c|c|c|c|}
\hline Ступень градиента & Время, мин & Доля элюента А, \% & Доля элюента Б, $\%$ \\
\hline Кондиционирование & 15 & 95 & 5 \\
\hline 0 & 0 & 95 & 5 \\
\hline 1 & 10 & 30 & 70 \\
\hline 2 & 20 & 30 & 70 \\
\hline
\end{tabular}

Условия хроматографического анализа. В качестве подвижной фазы А использовали смесь $0.5 \%$ раствора калия фосфорнокислого однозамещенного, метанола и триэтиламина в соотношении 74:25:1, доводили $\mathrm{pH}$ до 3.3 ортофосфорной кислотой. 
Подвижной фазой Б служил метанол с $\mathrm{pH} 3.3$, доведенным ортофосфорной кислотой. Режим элюирования - градиентный, условия представлены в таблице 1. Скорость потока подвижной фазы составляла $0.1 \mathrm{~cm}^{3} /$ мин, температура колонки $-35^{\circ} \mathrm{C}$, объем вводимой пробы - 20 мкл, длина волны УФ-детектирования - 260 нм.

\section{Обсуждение результатов}

Динамическое модифицирование подвижной фазы. Снижение предела количественного определения иматиниба в плазме крови и, как следствие, увеличение чувствительности методики по сравнению с ранее известными достигали улучшением важнейших хроматографических параметров пика иматиниба за счет динамического модифицирования подвижной фазы.

Обычно для большего удерживания сильнополярных соединений, за счет увеличения липофильности сорбата и, следовательно, обеспечения хорошей разделительной способности и емкости сорбента используют добавку неорганической соли в подвижную фазу [16]. Именно поэтому, при определении иматиниба, обладающего ярко выраженными основными свойствами, применяли в качестве подвижной фазы А $0.5 \%$ раствор калия дигидрофосфата с добавкой метанола (25\%). Для определения иматиниба в ионной форме разделение проводили при кислом значении $\mathrm{pH}$ элюентов А и Б равном 3.3. С целью дезактивации остаточных силанольных групп сорбента и предотвращения образования водородных связей с атомами азота иматиниба, приводящего к размыванию хроматографической зоны пика аналита $[2,5,7]$, в качестве модификатора использовали добавку триэтиламина (1 \%) в подвижную фазу А. Использование всех перечисленных модификаторов подвижной фазы привело к получению симметричного и узкого пика иматиниба на получаемых хроматограммах.

Выбор условий подготовки проб. В ранее известных работах в качестве пробоподготовки при извлечении иматиниба из биологической матрицы используют метод осаждения белков $[2,7-8,12,17]$ и твердофазную экстракцию (ТФЭ) [4-6, 910]. Обычно при осаждении белков не удается полноценно избавиться от эндогенных соединений плазмы крови (жиров, липидов и др.) и, следовательно, матричного эффекта, мешающего селективности определения, вследствие наличия множества пиков на хроматограмме аналита [7]. Использование метода жидкостно-жидкостной экстракции (ЖЖЭ) при анализе нескольких сотен, а иногда и тысяч образцов плазмы крови добровольцев в исследовании биоэквивалентности является более экономичным, чем применение картриджей ТФЭ. В связи с этим, нами был выбран метод жидкостно-жидкостной экстракции ацетонитрилом по принципу QuEChERS, основанный на извлечении аналита из матрицы ацетонитрилом, смешивающимся с водой в любых пропорциях, с последующим разделением водно-органического слоя за счет повышения ионной силы водной фазы с помощью неорганической соли [18]. В качестве неорганической соли использовали натрия хлорид, являющийся дешевым и доступным высаливателем, который при разделении фаз дополнительно денатурирует и осаждает белки плазмы крови. В отличие от метода осаждения белков, при экстракции ацетонитрилом происходит незначительный перенос эндогенных веществ плазмы крови в органический слой, что непременно повышает качество получаемых хроматограмм (наличие меньшего числа посторонних пиков) и как следствие - селективность.

Валидация методики. Валидацию разработанной методики количественного определения иматиниба в плазме крови человека проводили на основании «Руководства по экспертизе лекарственных средств. Том 1» (Россия, 2013) и согласно требованиям руководств по валидации биоаналитических методик «Guidance for Industry: 
Bioanalytical method validation» (FDA, США, 2001) и «Guideline on validation of bioanalytical methods» (EMA, Англия, 2009) по следующим характеристикам: селективность, предел обнаружения и нижний предел количественного определения, линейность, правильность и прецизионность, перенос, стабильность.

Селективность. С целью подтверждения селективности аналитической методики анализировали 6 образцов плазмы крови, не содержащих иматиниб (холостых образцов) и 6 образцов плазмы крови, содержащих иматиниб в концентрации $1680 \mathrm{Hг} / \mathrm{cm}^{3}$. Хроматограммы холостого образца плазмы крови и образца плазмы

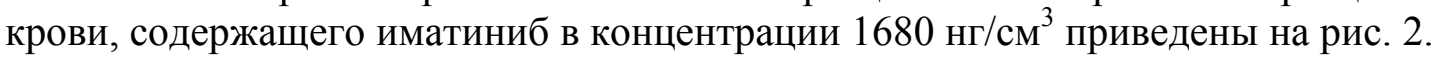

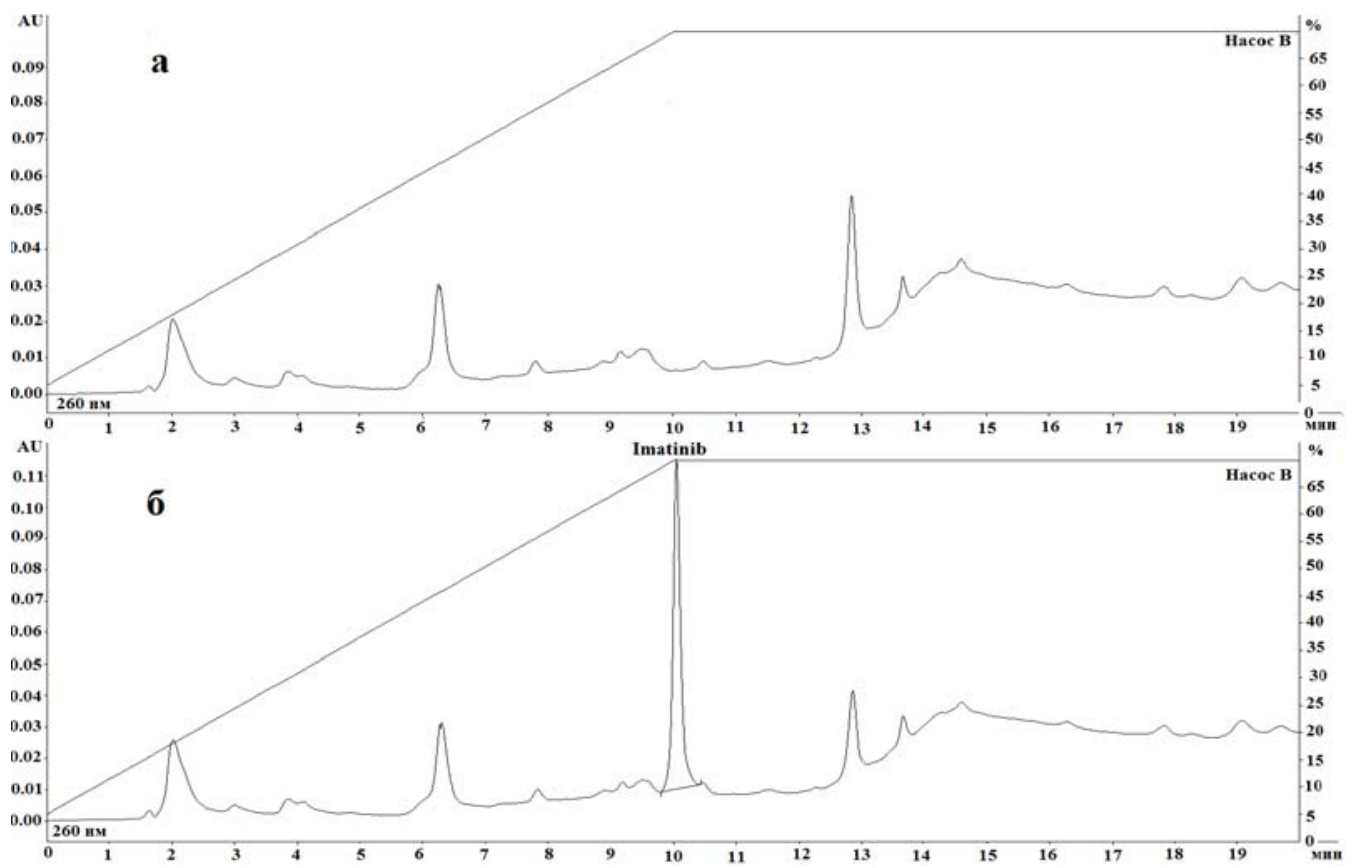

Рис. 2. Хроматограммы холостого образца плазмы крови (а) и образца плазмы крови, содержащего иматиниб в концентрации 1680 нг/ $\mathrm{cm}^{3}$ (б).

В ходе анализа выявлено отсутствие на хроматограммах холостых образцов пиков веществ, совпадающих по времени удерживания с пиком определяемого вещества - иматиниба.

Определены основные параметры пригодности хроматографической системы:

- время удерживания - 10.05 мин;

- эффективность колонки по пику иматиниба - 38606 ТТ;

- разрешение - 2.48;

- фактор асимметрии пика иматиниба - 1.3.

Предел обнаружения и нижний предел количественного определения. Для установления предела обнаружения и нижнего предела количественного определения (НПКО) использовали соотношение сигнал/шум, величины которых по требованиям должны составлять не менее 3:1 и 10:1 соответственно. Сравнивали величины сигналов, полученные для контрольного опыта (при отсутствии определяемого вещества в аналите), и для образцов с низкими концентрациями определяемого вещества. Установили, что пределом обнаружения иматиниба в плазме крови, согласно разработан-

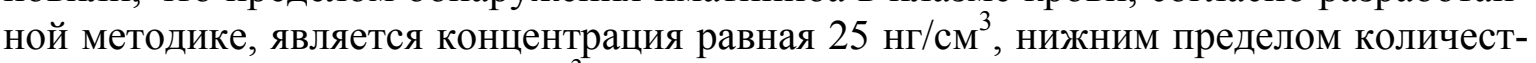
венного определения - $42 \mathrm{Hг} / \mathrm{cm}^{3}$.

Линейность градуировочного графика. Проводили анализ 7 образцов плазмы крови с концентрацией иматиниба: $42 \mathrm{Hг} / \mathrm{cm}^{3}, 420 \mathrm{Hг} / \mathrm{cm}^{3}, 840 \mathrm{Hг} / \mathrm{cm}^{3}, 1680 \mathrm{Hг} / \mathrm{cm}^{3}$, 


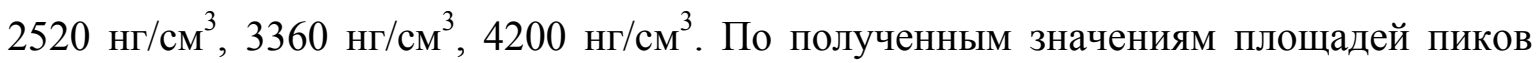
был построен градуировочный график, представленный на рис. 3.

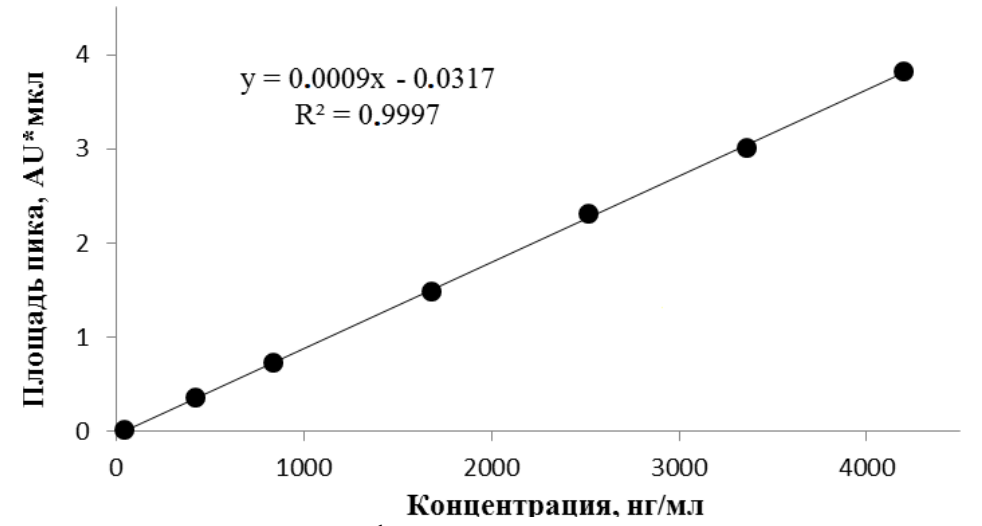

Рис. 3. Градуировочный график зависимости площади пика иматиниба от его концентрации в плазме крови

По полученной кривой (рис. 3) проводили обратный расчет концентраций градуировочных растворов. Отклонения концентраций градуировочных растворов, рассчитанных по графику, от фактических значений, приведены в таблице 2.

Таблица 2. Отклонения концентраций градуировочных растворов от фактических значений

\begin{tabular}{|c|c|c|c|c|c|c|c|}
\hline $\mathrm{C}$, факт, $\mathrm{Hг} / \mathrm{cm}^{3}$ & 42.0 & 420.0 & 840.0 & 1680.0 & 2520.0 & 3360.0 & 4200.0 \\
\hline $\begin{array}{c}\text { С рассчит, } \\
\mathrm{H} \Gamma \mathrm{cm}^{3}\end{array}$ & 49.9 & 432.9 & 824.6 & 1650.3 & 2563.3 & 3327.1 & 4213.9 \\
\hline$\delta, \%$ & 18.8 & 3.1 & 1.8 & 1.8 & 1.7 & 1.0 & 0.3 \\
\hline Норма, \% & Не более 20 & & & & 15 & & \\
\hline
\end{tabular}

Полученные отклонения соответствуют нормам - не более 20 \% для нижнего предела количественного определения и не более $15 \%$ - для остальных точек.

Правильность и прецизионность

Для оценки правильности и прецизионности в условиях повторяемости, а также внутрилабораторной прецизионности, анализировали образцы плазмы крови, содержащие иматиниб в 4 различных концентрациях, перекрывающих собой диапазон градуировочной кривой: НПКО, тройная величина НПКО, 50 \% диапазона градуировочной кривой и 80 \% от верхнего диапазона градуировочной кривой, рассчитывая при этом количественное содержание по градуировочному графику. Для полученных значений концентраций были рассчитаны величины относительного стандартного отклонения (RSD, \%) и относительной погрешности $(\delta, \%)$, приведенные в таблицах 3-4.

Полученные величины относительного стандартного отклонения и относительной погрешности соответствуют требованиям - не более $20 \%$ для нижнего предела количественного определения и не более $15 \%$ - для остальных точек.

Перенос. Перенос оценивался введением холостого образца после анализа образцов с концентрацией, соответствующей верхнему пределу количественного определения. На полученной хроматограмме холостого образца не обнаружено пика иматиниба, следовательно, не происходит переноса сигнала от определяемого вещества в холостой образец после анализа образца с высокой концентрацией. 
Таблица 3. Правильность и прецизионность в условиях повторяемости

\begin{tabular}{|c|c|c|c|c|}
\hline 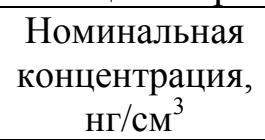 & $\begin{array}{c}\text { Рассчитанная } \\
\text { концентрация, } \\
\mathrm{Hг} / \mathrm{cm}^{3}\end{array}$ & 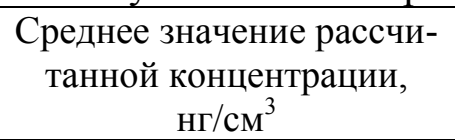 & RSD, \% & $\delta, \%$ \\
\hline \multirow{5}{*}{42} & 42 & \multirow[t]{5}{*}{ 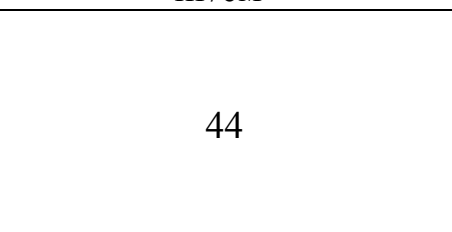 } & \multirow{5}{*}{2.6} & \multirow{5}{*}{4.7} \\
\hline & 44 & & & \\
\hline & 44 & & & \\
\hline & 43 & & & \\
\hline & 45 & & & \\
\hline \multirow{5}{*}{126} & 118 & \multirow{5}{*}{116} & \multirow{5}{*}{2.9} & \multirow{5}{*}{7.9} \\
\hline & 118 & & & \\
\hline & 118 & & & \\
\hline & 113 & & & \\
\hline & 111 & & & \\
\hline \multirow{5}{*}{2100} & 2180 & \multirow{5}{*}{2040} & \multirow{5}{*}{7.4} & \multirow{5}{*}{2.9} \\
\hline & 2186 & & & \\
\hline & 1843 & & & \\
\hline & 2056 & & & \\
\hline & 1933 & & & \\
\hline \multirow{5}{*}{3360} & 3425 & \multirow{5}{*}{3498} & \multirow{5}{*}{4.2} & \multirow{5}{*}{4.1} \\
\hline & 3420 & & & \\
\hline & 3340 & & & \\
\hline & 3668 & & & \\
\hline & 3638 & & & \\
\hline
\end{tabular}

Таблица 4. Внутрилабораторная прецизионность

\begin{tabular}{|c|c|c|c|}
\hline $\begin{array}{c}\text { Номинальная концентра- } \\
\text { ция, } \mathrm{Hг} / \mathrm{cm}^{3}\end{array}$ & $\begin{array}{c}\text { Рассчитанная } \\
\text { концентрация, } \\
\mathrm{Hг} / \mathrm{cm}^{3} \\
\end{array}$ & 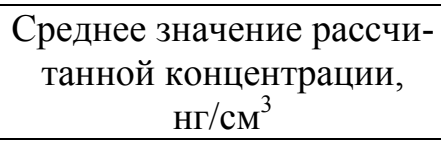 & RSD, $\%$ \\
\hline \multirow{5}{*}{42} & 42 & \multirow{5}{*}{ 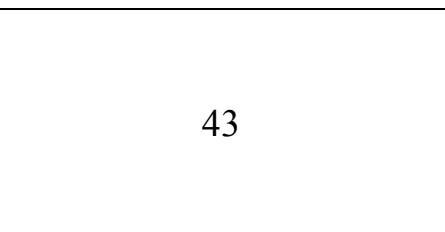 } & \multirow{5}{*}{2.1} \\
\hline & 44 & & \\
\hline & 43 & & \\
\hline & 44 & & \\
\hline & 44 & & \\
\hline \multirow{5}{*}{126} & 113 & \multirow{5}{*}{118} & \multirow{5}{*}{2.5} \\
\hline & 117 & & \\
\hline & 119 & & \\
\hline & 121 & & \\
\hline & 118 & & \\
\hline \multirow{5}{*}{2100} & 2143 & \multirow{5}{*}{2081} & \multirow{5}{*}{5.6} \\
\hline & 1916 & & \\
\hline & 2021 & & \\
\hline & 2219 & & \\
\hline & 2107 & & \\
\hline \multirow{5}{*}{3360} & 3563 & \multirow{5}{*}{3547} & \multirow{5}{*}{5.4} \\
\hline & 3213 & & \\
\hline & 3678 & & \\
\hline & 3657 & & \\
\hline & 3626 & & \\
\hline
\end{tabular}

Стабильность. Оценку стабильности проводили на образцах плазмы крови,

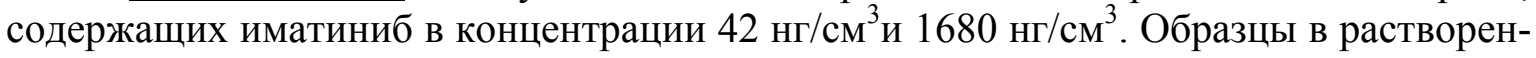


ном виде и в виде сухих остатков были помещены в различные условия хранения: при комнатной температуре, в холодильник $\left(+3-6^{\circ} \mathrm{C}\right)$, в морозильную камеру $(-18-$ $\left.24^{\circ} \mathrm{C}\right)$. Образцы анализировали через 24 часа. Также модельные образцы плазмы крови, содержащие иматиниб, были подвергнуты многократному (3 цикла) размораживанию и замораживанию в течение 12 часов с последующим анализом. Свежеприготовленные образцы из той же серии анализировали сразу после приготовления.

При изучении стабильности было выявлено, что отклонения полученных значений концентраций варьируется в пределах от 0.42 до $9.40 \%$ и соответствуют требованиям (не более 10\%), что свидетельствует о стабильности образцов, содержащих иматиниб, в течение 24 часов при хранении в холодильнике и при комнатной температуре, а также при многократном замораживании и размораживании.

\section{Заключение}

Разработана новая высокочувствительная аналитическая методика количественного определения иматиниба в плазме крови человека методом ВЭЖХ с УФдетектированием, подобраны оптимальные условия пробоподготовки для извлечения иматиниба из биологической матрицы. Подтверждена пригодность разработанной методики путем ее валидации, доказаны селективность, линейность, правильность и прецизионность. Разработанная методика может быть использована для количественной оценки содержания иматиниба в плазме крови человека при различных фармакокинетических исследованиях и клиническом исследовании биоэквивалентности.

\section{Список литературы}

1. Абдулкадыров К.М., Шуваев В.А., Фоминых М.С. // Клиническая онкогематология. Фундаментальные исследования и клиническая практика. 2014. Т. 7. № 3. С. 311316.

2. Медведев Ю.В., Шохин Е.И., Савченко А.Ю., Ярушок Т.А. // Разработка и регистрачия лекарственных средств. 2013. № 1(2). C. 74-77.

3. Шохин И.Е., Раменская Г.В., Медведев Ю.В., Ярушок Т.А. и др. // Сорбиионные и хроматографические прочессы. 2013. Т. 13. № 2. C. 220-228.

4. Miura M., Takahashi N., Sawada K. // Journal of Chromatographic Science. 2011. Vol. 49, pp. 412-415.

5. Pirro E., De Francia S., De Martino F., Fava C. et al. // Journal of Chromatographic Science. 2011. Vol. 49, pp. 753-757.

6. Davies A., Hayes A.K., Knight K., Watmough S.J. et al. // Leukemia Research. 2010. Vol. 34, pp. 702-707.

7. Roth O., Spreux-Varoquaux O., Bouchet S., Rousselot P. et al. // Clinica Chimica Acta. 2010. Vol. 411. pp. 140-146.
8. Teoh M., Narayanan P., Moo K.S., Radhakrisman S. et al. // Pakistan Journal of Pharmaceutical Sciences. 2010. Vol. 23. pp. 35-41.

9. Neville K., Parise R.A., Thompson P., Aleksic A. et al. // Clinical Cancer Research. 2004. Vol. 10, pp. 2525-2529.

10. Dziadosz M., Bartels H. // Acta Chimica Slovenica. 2011. Vol. 58. pp. 347-350.

11. Golabchifar A.A., Rouini M.R., Shafaghi B., Rezaee S. et al. // Talanta. 2011. Vol. 85. pp. 2320-2329.

12. De Francia S., D'Avolio A., De Martino F., Pirro E. et al. // Journal of Chromatography B. 2009. Vol. 877. pp. 1721-1726.

13. Francis J., Dubashi B., Sundaram R., Pradhan S.C. et al. // World Journal of Pharmaceutical Research. 2014. Vol. 3. pp. 1067-1075.

14. Yang J.S., Cho E.G., Huh W., Ko JW. et al. // Bulletin of the Korean Chemical Society. 2013. Vol. 34. pp. 2425-2430. DOI: 10.5012/bkcs.2013.34.8.2425

15. Lankheet N.A.G., Hillebrand M.J.X., Rosing H., Schellens J.H.M. et al. // Biomedical Chromatography. 2013. Vol. 27. No 4. pp. 466476. DOI: $10.1002 / \mathrm{bmc} .2814$ 
16. Сапрыкин Л.В. // Химический анализ. 2005. C. 20-36.

17. Shetty R., Kini S., Musmade P., Theerthahalli A. et al. // Pharmacologyonline. 2008. Vol. 3.pp. 752-760.

\section{References}

1. Abdulkadyrov K.M., Shuvaev V.A., Fominyh M.S.,/ Klinicheskaya onkogematologiya. Fundamental'nye issledovaniya i klinicheskaya praktika, 2014, Vol. 7, No 3, pp. 311-316.

2. Medvedev Ju.V., Shohin E.I., Savchenko A.Ju., Jarushok T.A., Razrabotka i registracija lekarstvennyh sredstv, 2013, No 1(2), pp. 74-77.

3. Shohin I.E., Ramenskaja G.V., Medvedev Ju.V., Jarushok T.A. et al., Sorbtsionnye $i$ khromatograficheskie protsessy, 2013, Vol. 13, No 2, pp. 220-228.

4. Miura M., Takahashi N., Sawada K., J. of Chromatographic Science, 2011, Vol. 49, pp. 412-415. DOI: 10.1093/chromsci/49.5.412

5. Pirro E., De Francia S., De Martino F., Fava C. et al., J. of Chromatographic Science, 2011, Vol. 49, pp. 753-757. DOI: $10.1093 / \mathrm{chrsci} / 49.10 .753$

6. Davies A., Hayes A.K., Knight K., Watmough S.J. et al., Leukemia Research, 2010, Vol. 34, pp. 702-707. DOI:10.1016/j.leukres.2009.11.009

7. Roth O., Spreux-Varoquaux O., Bouchet S., Rousselot P. et al., Clinica Chimica Acta, 2010, Vol. 411, pp. 140-146. DOI: 10.1016/j.cca.2009.10.007

8. Teoh M., Narayanan P., Moo K.S., Radhakrisman S. et al., Pakistan Journal of Pharmaceutical Sciences, 2010, Vol. 23, pp. 35-41.

9. Neville K., Parise R.A., Thompson P., Aleksic A. et al., Clinical Cancer Research,

Леонов Клим Андреевич - аспирант кафедры физической и аналитической химии, Национальный исследовательский Томский политехнический университет, Томск

Бакибаев Абдигали Абдиманапович - профессор, д.Х.н., ведущий научный сотрудник лаборатории органического синтеза химического факультета, Национальный исследовательский Томский государственный университет, Томск
18. Anzillotti L., Odoardi S., Rossi S.S. // Forensic Science Int. 2014. Vol. 243, pp. 99106. DOI: $10.1016 /$ j.forsciint.2014.05.005

2004, Vol. 10, pp. 2525-2529. DOI: $10.1016 /$ j.clpt.2003.11.223

10. Dziadosz M., Bartels H., Acta Chimica Slovenica, 2011, Vol. 58, pp. 347-350.

11.Golabchifar A.A., Rouini M.R., Shafaghi B., Rezaee S. et al., Talanta, 2011, Vol. 85, pp. 2320-2329. DOI: 10.1016/j.talanta.2011.07.093

12.De Francia S., D'Avolio A., De Martino F., Pirro E. et al., J. of Chromatography B, 2009, Vol. 877, pp. 1721-1726.

DOI: $10.1016 /$ j.jchromb.2009.04.028

13. Francis J., Dubashi B., Sundaram R., Pradhan S.C. et al., World J. of Pharmaceutical Research, 2014, Vol. 3, pp. 1067-1075.

14. Yang J.S., Cho E.G., Huh W., Ko J-W et al., Bulletin of the Korean Chemical Society, 2013, Vol. 34, pp. 2425-2430. DOI: 10.5012/bkcs.2013.34.8.2425

15.Lankheet N.A.G., Hillebrand M.J.X., Rosing H., Schellens J.H.M. et al., Biomedical Chromatography, 2013,Vol. 27, No 4, pp. 466476. DOI: $10.1002 / \mathrm{bmc} .2814$

16. Saprykin L.V., Himicheskij analiz, 2005, pp. 20-36.

17. Shetty R., Kini S., Musmade P., Theerthahalli A. et al., Pharmacologyonline, 2008, Vol. 3, pp. 752-760.

18.Anzillotti L., Odoardi S., Rossi S.S., Forensic Science Int., 2014, Vol. 243,

pp. 99-106.

DOI: 10.1016/j.forsciint.2014.05.005

Leonov Klim A. - PhD student of Department of Physical and Analytical Chemistry, National Research Tomsk Polytechnic University, Tomsk, e-mail: leonov_k90@mail.ru

Bakibaev Abdigali A. - Professor, Doctor of Chemistry, Senior Researcher in Laboratory of organic synthesis Faculty of Chemistry, National Research Tomsk State University, Tomsk 